\title{
Nasolabial symmetry and esthetics in cleft lip and palate: analysis of 3D facial images
}

\author{
Dries J. Desmedt • Thomas J. Maal • Mette A. Kuijpers • \\ Ewald M. Bronkhorst • Anne Marie Kuijpers-Jagtman • \\ Piotr S. Fudalej
}

Received: 9 May 2014 / Accepted: 3 March 2015 / Published online: 24 March 2015

(C) Springer-Verlag Berlin Heidelberg 2015

\begin{abstract}
Objectives To determine the relationship between nasolabial symmetry and esthetics in subjects with orofacial clefts.

Material and methods Eighty-four subjects (mean age 10 years, standard deviation 1.5 ) with various types of nonsyndromic clefts were included: 11 had unilateral cleft lip (UCL); 30 had unilateral cleft lip and alveolus (UCLA); and 43 had unilateral cleft lip, alveolus, and palate (UCLAP). A 3D stereophotogrammetric image of the face was taken for each subject. Symmetry and esthetics were evaluated on cropped 3D facial images. The degree of asymmetry of the nasolabial area was calculated based on all 3D data points using a surface registration algorithm. Esthetic ratings of various elements of nasal morphology were performed by eight
\end{abstract}

D. J. Desmedt $\cdot$ M. A. Kuijpers · A. M. Kuijpers-Jagtman Department of Orthodontics and Craniofacial Biology, Radboud University Medical Center, Nijmegen, The Netherlands

T. J. Maal

Department of Maxillofacial Surgery, Radboud University Medical Center, Nijmegen, The Netherlands

M. A. Kuijpers · A. M. Kuijpers-Jagtman

Cleft Palate Craniofacial Unit, Radboud University Medical Center, Nijmegen, The Netherlands

\section{E. M. Bronkhorst}

Department of Preventive and Curative Dentistry, Radboud

University Medical Center, Nijmegen, The Netherlands

\section{P. S. Fudalej}

Department of Orthodontics and Dentofacial Orthopedics, University of Bern, Bern, Switzerland

P. S. Fudalej $(\square)$

Department of Orthodontics, Palacky University Olomouc,

Olomouc, Czech Republic

e-mail: piotr.fudalej@zmk.unibe.ch lay raters on a $100 \mathrm{~mm}$ visual analog scale. Statistical analysis included ANOVA tests and regression models.

Results Nasolabial asymmetry increased with growing severity of the cleft $(p=0.029)$. Overall, nasolabial appearance was affected by nasolabial asymmetry; subjects with more nasolabial asymmetry were judged as having a less esthetically pleasing nasolabial area $(p<0.001)$. However, the relationship between nasolabial symmetry and esthetics was relatively weak in subjects with UCLAP, in whom only vermilion border esthetics was associated with asymmetry.

Conclusions Nasolabial symmetry assessed with 3D facial imaging can be used as an objective measure of treatment outcome in subjects with less severe cleft deformity. In subjects with more severe cleft types, other factors may play a decisive role.

Clinical significance Assessment of nasolabial symmetry is a useful measure of treatment success in less severe cleft types.

Keywords Cleft lip $\cdot$ Cleft palate $\cdot$ Esthetics $\cdot$ Face . Morphology $\cdot$ Imaging $\cdot$ Three dimensional

\section{Introduction}

Facial appearance plays an important role in social interactions in children, teenagers, and adults. Attractive faces activate reward centers in the brain $[1,2]$, and they elicit positive personality attributions according to the "what is beautiful is good" stereotype [3, 4]. Facial attractiveness has also been demonstrated to influence initial impressions [5], helping behavior [6], and teacher judgments of student intelligence and future academic potential [7]. These effects are robust and extend beyond initial impressions of strangers to long-term interactions with people [4]. 
For many years, facial attractiveness was thought to reflect arbitrary standards of beauty set by cultures. However, several observations suggest that esthetic preferences may be a part of biological rather than cultural heritage. For example, (1) people from different cultures generally agree on which faces are attractive, (2) men and women agree on attractiveness [4, 8-11], and (3) preferences for facial attractiveness emerge early in development, before cultural standards of beauty are likely to be assimilated [12-17]. These data suggest that esthetic preferences might be by-products of the way the brain processes information [18-20]. A possible mechanism is that the faster the perceiver can process an object (i.e., decode the information included in the object), the more positive his/her esthetic response. One of the features that facilitate fluent processing is symmetry. In principle, symmetric shapes contain less information than asymmetric shapes; hence, they are easier to process and are thus more pleasing [8]. Experimental studies have confirmed that perfectly symmetric faces are more attractive than the original, slightly asymmetric faces $[21,22]$.

In patients with cleft lip and palate (CLP), one of the treatment goals is to minimize asymmetry of the nasolabial area in order to improve facial appearance. However, the association between nasolabial symmetry and facial esthetics in patients with CLP is not clear. Some authors found correlations between esthetic ratings and objectively measured nasolabial symmetry [23-25], whereas others did not [26, 27]. A possible reason for these conflicting findings is that symmetry and esthetics were assessed primarily on 2D nasolabial images [24, 25, 27]. Although Russell et al. [23] and Russell and Tompson [26] used nose casts for the evaluation of symmetry, they performed relatively few measurements, which may not have reflected the true nasolabial asymmetry. Moreover, the esthetics of the nasolabial area should be assessed on a 3D stimulus, as the face is a $3 \mathrm{D}$ structure. Therefore, the aim of this study was to evaluate the association between nasolabial symmetry and esthetics assessed on 3D photogrammetric images in a sample of preadolescent children with various types of CLP: (1) unilateral cleft lip (UCL); (2) unilateral cleft lip and alveolus (UCLA); and (3) unilateral cleft lip, alveolus, and palate (UCLAP). Our research hypothesis was that there is a relationship between the symmetry and the esthetics of the nasolabial region.

\section{Materials and methods}

\section{Subjects}

The files of the Cleft Palate Craniofacial Unit at the Radboud University Medical Center, Nijmegen, The Netherlands were searched to identify all patients meeting the following inclusion criteria: (1) diagnosis of nonsyndromic UCL, UCLA, or UCLAP, ascertained by the clinical geneticist of the team and
(2) available 3D stereophotogrammetric images of the face taken at approximately 10 years of age.

The patient group consisted of 84 patients with a mean age of 10 years (range: 8.6-12.3 years). Eleven of the patients presented with UCL, 30 had UCLA, and 43 had UCLAP. All patients were treated in accordance with the standardized protocols used at the unit.

The use of anonymous data gathered during routine patient care is in accordance with Dutch law on medical research. A written statement of the institutional review board (IRB) was obtained stating that this study does not fall within the remit of the Medical Research Involving Human Subjects Act (WMO). Therefore, the present investigation could be carried out without an individual approval by an accredited research ethics committee. No formal waiver of approval by the IRB was obtained. All parents/legal guardians gave written informed consent for the use of images of their children in the study.

Three-dimensional photographs of all patients were taken with the same stereophotogrammetrical camera setup (3dMD face System; 3dMD LLC, Atlanta, GA, USA) under standardized conditions. Patients were positioned in the natural head position and asked to keep their eyes open and to relax their facial musculature. All images were taken by an experienced photographer. Based on the 3D images, (1) mean absolute nasolabial asymmetry and (2) nasolabial esthetics were assessed.

\section{Calculation of mean absolute nasolabial asymmetry}

The four-step method to quantify soft-tissue facial asymmetry described by Verhoeven et al. was applied in this study [28]. Because the original method evaluates facial asymmetry and the aim of this study was to quantify nasolabial asymmetry, the method was supplemented with additional steps. The additional steps are described below.

Positioning Maintaining the line through the right and left exocanthi horizontal, the head was rotated until the line passing through the exocanthion-superaurale became horizontal. A midpupillar point (positioned on the crossing of the midline of the nose and the bipupilar line) was then placed. This was necessary to create a $3 \mathrm{D}$ positioning of the head with the sagittal, coronal, and transversal planes indicated.

Cropping In order to define the area to be cropped, the following points were placed on the $3 \mathrm{D}$ images: right and left endocanthion, right and left exocanthion, right and left stomion, and the point indicating the lowest position on the curvature of the labrale inferius (Fig. 1).

We created limiting planes passing through (A) the left endocanthion and left stomion, perpendicular to the sagittal pane; (B) the right endocanthion and right stomion, perpendicular to the sagittal plane; $(\mathrm{C})$ the right and left exocanthion, 
Fig. 1 Nasolabial area used for analysis. 1 exocanthion right, 2 endocanthion right, 3 midpupillar point, 4 endocanthion left, 5 exocanthion left, 6 stomion right, 7 lowest position on the curvature of labraleinferius, and 8 stomion left. The following areas were created: $A$ left endocanthion and left stomion, perpendicular to the sagittal pane; $B$ right endocanthion and right stomion, perpendicular to the sagittal plane; $C$ right and left exocanthion, perpendicular to the transverse plane; and $D$ lowest point of labraleinferius, parallel to the plane passing through both exocanthi. Yellow area is defined as the nasolabial area

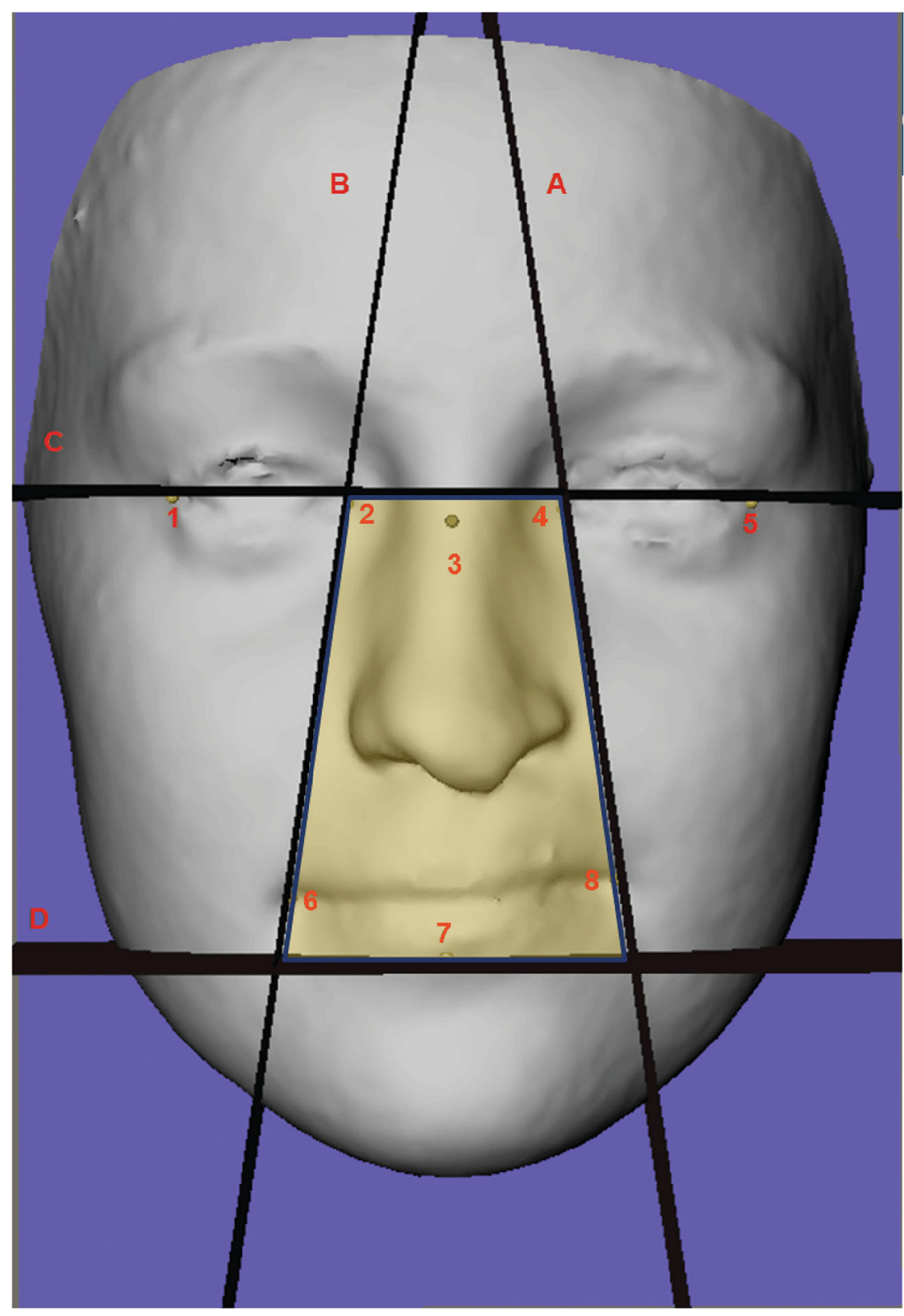

perpendicular to the transverse plane; and (D) the lowest point of the labraleinferius, parallel to the plane passing through both exocanthi (Fig. 1). The area confined within these four planes was defined as the nasolabial area (Fig. 1). Subsequently, a distance map of this area was calculated (Fig. 2). Two authors performed the analysis of nasolabial asymmetry: DD analyzed all images, whereas YC analyzed 25 randomly selected images to determine inter-rater reliability.

\section{Assessment of nasolabial esthetics}

Eight postgraduate students (four men and four women; age range: 25-31 years) from the Department of Orthodontics and Craniofacial Biology at Radboud UMC who had no experience with CLP treatment were asked to rate nasolabial esthetics on $3 \mathrm{D}$ images with the modified Asher-McDade esthetic index [29]. According to the original index, four elements of nasolabial morphology - nasal form, nasal deviation, vermilion border, and nasolabial profile - are assessed on a five-point scale (1 corresponds with the best, 5 with the worst outcome). In the present study, a $100 \mathrm{~mm}$ visual analog scale (VAS) instead of a five-point scale was used. The overall esthetic score was calculated as the mean of scores assigned to four nasolabial components. In the VAS scale, $0 \mathrm{~mm}$ corresponded with the least and $100 \mathrm{~mm}$ with the most esthetic outcome. The raters evaluated the cropped 3D images on their own laptops (see Fig. 3). They could manipulate the pictures in all directions, and there was no time limit for scoring. All raters gave informed consent. 
Fig. 2 Distance map of the nasolabial segment. Red color represents surface points that lie behind the surface of the mirrored image (indicated with a negative number, scale maximum is $-5 \mathrm{~mm}$ ) and green color represents surface points that lie in front of the mirrored image (indicated with a positive number, scale maximum is $+5 \mathrm{~mm}$ )

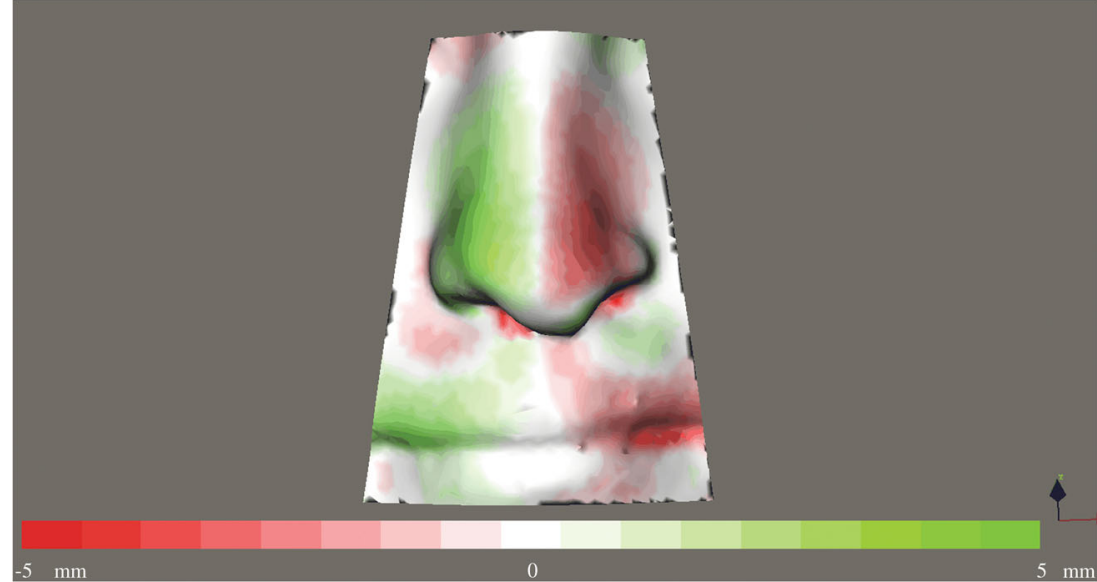

In order to determine intra-rater reliability, two rating sessions were carried out, with a 1 month interval. During the first rating session, 84 patients were rated, and during the second, 35 randomly selected patients from the first rating session were re-rated.

\section{Statistical analysis}

Error of the method of the evaluation of nasolabial asymmetry was assessed by computing duplicate measurement errors (DMEs), reliability coefficients, and differences between the

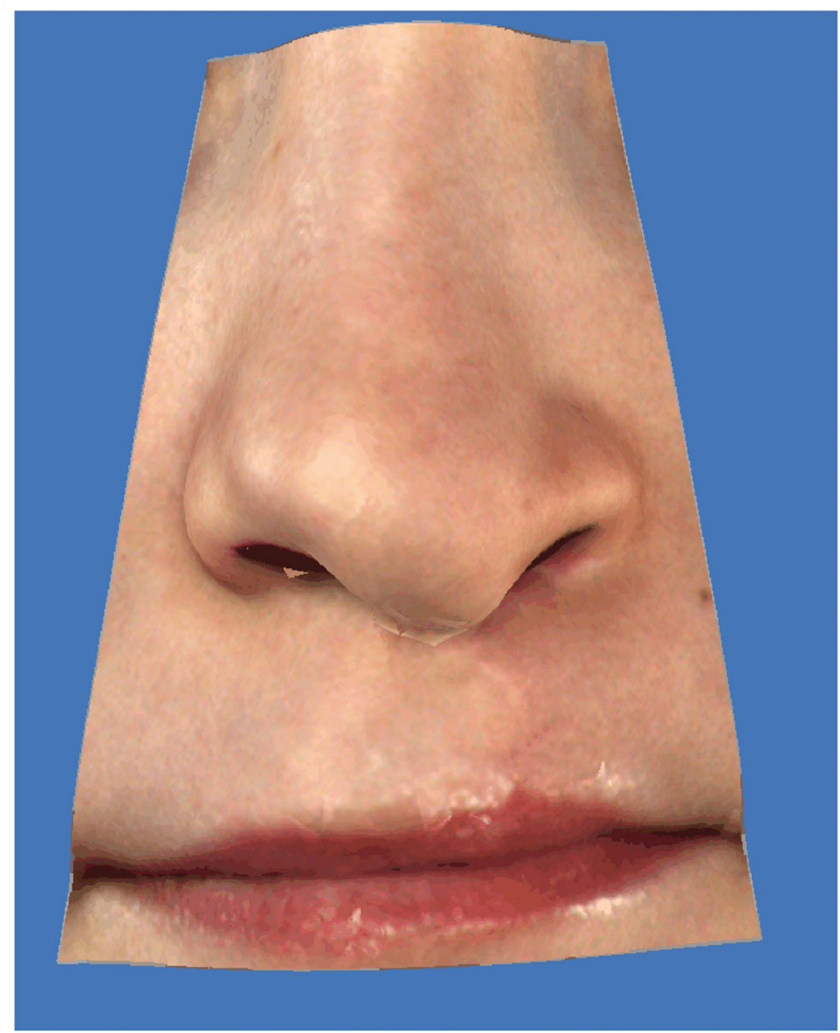

Fig. 3 A 3D nasolabial image used for esthetic rating. Judges were free to manipulate the image in all directions means. Paired $t$ tests were run to assess if there were systematic errors among observers. To evaluate whether there was a difference in the mean absolute asymmetry among the UCL, UCLA, and UCLAP groups, analysis of variance (ANOVA) was performed.

Judgment of esthetics can produce considerable variation of scores among raters $[29,30]$. The scores for different observers can be averaged for each individual nasolabial component (nasal form, nasal deviation, vermilion border, and nasolabial profile), provided that there is sufficient coherence among the observers. Cronbach's alpha reliability coefficient [31] was calculated for each individual component to evaluate the inter-rater coherence. If an inter-observer coherence was adequate (Cronbach's alpha $>0.80$ ) [32], the mean scores of the eight observers were used in the "Results" section. To test the reproducibility of the esthetic rating, a paired $t$ test was performed using the mean scores of the eight observers from the first and second rating sessions. To test if there was a difference between the mean esthetic score for the different cleft types, an ANOVA was run.

Regression analyses were performed to investigate an association between the mean absolute nasolabial asymmetry (independent variable) and nasolabial esthetic scores overall and for four components of nasolabial morphology (dependent variables). All statistical analyses were done with SPSS software (version 20).

\section{Results}

Method reliability

Analysis of the measurement quality of the absolute nasolabial asymmetry was satisfactory. The inter-rater DME was $0.05 \mathrm{~mm}$, the reliability coefficient was 0.99 , and the difference in the means between the observations was $0.008 \mathrm{~mm}$ ( $95 \% \mathrm{CI}$ 0.019 to 0.035$)$. There was no systematic difference $(p=0.545)$. 
The values of Cronbach's alpha coefficients ranged from 0.83 to 0.91 , which indicated good coherence among the eight observers. As a result, the average scores of all observers were used in further reliability analyses (Table 1). For three of the four nasolabial components, there was a systematic difference between the scores from the two sessions. However, the difference was relatively small.

\section{Absolute nasolabial asymmetry}

The mean absolute nasolabial asymmetry ranged from 0.75 to $1.14 \mathrm{~mm}$ and displayed increasing asymmetry with increasing severity of cleft type (Table 2). ANOVA tests showed significant differences among the three groups $(p=0.029)$. Post hoc Tukey tests demonstrated a statistically significant difference between the UCLAP and UCL groups ( $p=0.022)$ (Table 3 ).

Nasolabial esthetics

Overall esthetic score The mean overall esthetic scores ranged from 59.40 to 50.53 and implied decreasing esthetics with increasing cleft severity (Table 4). ANOVA with post hoc Tukey tests demonstrated statistically significant differences between the UCL and UCLAP groups $(p=0.018)$ and between the UCLA and UCLAP groups ( $p=0.033$ ) (Table 5).

Esthetic scores of nasolabial components The mean esthetic scores for the nasolabial components in the UCL, UCLA, and UCLAP groups are shown in Table 4. ANOVA with post hoc Tukey tests demonstrated that the esthetics of nose shape were different between the UCL and UCLAP groups $(p=0.04)$, whereas the esthetics of nose deviation were different between the UCL and UCLAP groups $(p=0.037)$ and between the UCLA and UCLAP groups $(p=0.039)$. The vermilion border and profile view had comparable esthetics in the various cleft type groups.

Association between nasolabial esthetics and absolute nasolabial asymmetry irrespective of cleft type

Table 6 presents the results of the regression analyses, with mean absolute nasolabial asymmetry as the independent var- iable and esthetic scores as dependent variables. Both asymmetry and esthetics were evaluated in a sample of 84 subjects that comprised all cleft types. In the pooled group, nasolabial asymmetry was associated with the esthetics of all nasolabial components. However, with the exception of nasolabial profile, the amount of variance of esthetic scores was explained relatively poorly by nasolabial asymmetry $\left(R^{2}\right.$ ranged from 0.11 to 0.25 ).

Association between nasolabial esthetics and absolute nasolabial asymmetry with respect to cleft type

In general, subjects with less severe cleft types (UCL and UCLA) showed a tendency toward a stronger association between nasolabial asymmetry and esthetics than subjects with the more severe cleft type (UCLAP) (Table 7). The esthetics of nasal shape and nose deviation were related to nasolabial asymmetry in the UCL and UCLA groups only. For vermilion border, the esthetic score was associated with nasolabial asymmetry in the UCLAP group only, whereas for the profile view, the esthetic score was related to nasolabial asymmetry in subjects from the UCL group. Overall, nasolabial esthetics was associated with nasolabial asymmetry in the UCL and UCLA groups only.

\section{Discussion}

One of the primary goals in the treatment of patients with CLP is to restore the anatomy of the orofacial region to create a good facial appearance. If treatment outcome is poor, psychological problems may develop [33]. However, assessment of facial esthetics in CLP is burdened with methodological difficulties, such as the subjectivity of judgment and the choice of raters (lay vs. professional). An evaluation of facial symmetry with 3D imaging techniques can aid in esthetic assessment. The rationale is that in subjects without a cleft anomaly, a symmetric face is regarded as more esthetic than an asymmetric face. Because this hypothesis has not been proven in CLP subjects, our objective was to assess the
Table 1 Reliability of nasolabial esthetic assessment

Bold font denotes statistical significance

$D M E$ duplicate measurement error, $C I$ confidence interval

\begin{tabular}{llllll}
\hline Nasolabial component & Reliability coefficient & DME & $\begin{array}{l}\text { Difference between } \\
\text { the means }\end{array}$ & $p$ & $95 \%$ CI \\
\hline Nose shape & 0.825 & 5.32 & 0.79 & 0.539 & -1.80 to 3.38 \\
Nose deviation & 0.761 & 5.14 & -2.69 & $\mathbf{0 . 0 3 5}$ & -5.19 to -0.20 \\
Vermilion border & 0.818 & 4.57 & 2.76 & $\mathbf{0 . 0 1 6}$ & 0.54 to 4.98 \\
Profile & 0.805 & 4.94 & -3.70 & $\mathbf{0 . 0 4 0}$ & -6.10 to -1.30 \\
\hline
\end{tabular}


Table 2 Mean absolute nasolabial asymmetry, expressed in $\mathrm{mm}$

\begin{tabular}{llll}
\hline Groups & Mean & SE & $95 \%$ CI \\
\hline UCL & 0.75 & 0.12 & 0.49 to 1.01 \\
UCLA & 1.04 & 0.77 & 0.88 to 1.20 \\
UCLP & 1.14 & 0.69 & 1.01 to 1.28 \\
\hline
\end{tabular}

$S E$ standard error, $C I$ confidence interval, ULC unilateral cleft lip, UCLA unilateral cleft lip and alveolus, UCLAP unilateral cleft lip and alveolus

association between nasolabial symmetry and esthetics in preadolescent children with various types of cleft.

Our findings demonstrate that, overall, absolute nasolabial asymmetry is associated with esthetics and can be used as an outcome measure in CLP. In the present study, subjects with more nasolabial asymmetry were judged to have a less esthetically pleasing nasolabial region, whereas subjects with less asymmetry of the nose and upper lip were rated as having a better nasolabial appearance. Other studies in which nasolabial symmetry and esthetics were evaluated on 3D images $[23,34]$ yielded ambiguous results. On one hand, the findings of Russell et al. (2001) showed that in subjects with UCLP, nasal symmetry and esthetics were weakly associated [23]. The authors evaluated nasal symmetry on plaster casts of the nose. Parameters that were measured were nasal area, perimeter, centroid, moments of area about the major and minor axes, angle to principal axis, anisometry, bulkiness (i.e., irregularity of the nasal shape), lateral offset, and projected and true inter-nostril angles. Of these variables, only perimeter and bulkiness showed positive correlations with esthetics of the nose. A possible explanation for the difference between the results of our study and those of Russell et al. is in the methodology. We calculated the distance map, which is a global measure of asymmetry, whereas Russell et al. used several nasal measurements, which reflect local asymmetry only. On the other hand, the findings

Table 3 Results of comparison of absolute nasolabial asymmetry in $\operatorname{UCL}(N=11)$, UCLA $(N=30)$, and UCLAP $(N=43)$ groups assessed with ANOVA and post hoc Tukey tests

\begin{tabular}{lllll}
\hline Groups & $\begin{array}{l}\text { Mean difference } \\
\text { between groups } \\
\text { (in mm) }\end{array}$ & SE & $p$ & $95 \%$ CI \\
\hline UCL vs. UCLA & 0.29 & 0.152 & 0.141 & -0.07 to 0.66 \\
UCL vs. UCLAP & 0.40 & 0.146 & $\mathbf{0 . 0 2 2}$ & 0.05 to 0.74 \\
UCLA vs. UCLAP & 0.10 & 0.103 & 0.575 & -0.14 to 0.35 \\
\hline
\end{tabular}

Bold font denotes statistical significance

$S E$, standard error; $C I$, confidence interval; $U C L$, unilateral cleft lip; $U C L A$, unilateral cleft lip and alveolus; UCLAP, unilateral cleft lip, alveolus, and palate of Meyer-Marcotty et al. (2010), who used a 3D imaging method comparable to ours, are in keeping with our results [34]. The authors found that the greater the facial asymmetry near the midline of the face, the more negative the perception of the facial appearance in adults with UCLAP.

Analysis of the association of nasolabial symmetry and esthetics with respect to cleft type (i.e., UCL, UCLA, and UCLAP) demonstrated that in subjects with the most severe cleft type (UCLAP), esthetic ratings were weakly correlated with the degree of asymmetry. Of the four assessed nasolabial components, only the perception of the vermilion border was affected by symmetry. This could be explained by the fact that a poor nasal form, frequently present in children with repaired UCLAP, could have a dominant effect on perception of the nasolabial appearance during esthetic evaluation. Earlier studies showed that obtaining a good nasal shape remains a challenge for the surgeon. The results of the Eurocleft study [30], the Warsaw-Nijmegen comparison [35], and the Americleft study [36] demonstrated that nasal shape receives more negative esthetic scores than other nasolabial components. This implies that creating normal nasal morphology in children with UCLAP is difficult and that many of them present with poor nasal form. Although we did not assess the extent to which nasal form in UCLAP deviates from the norm, it is likely that many subjects with UCLAP had an abnormally shaped nose and that this feature affected the raters' perception more than nasal asymmetry. Evidence supporting this hypothesis was presented by Meyer-Marcotty et al. [34]. The authors compared facial symmetry and esthetics between adults with cleft lip and palate and subjects surgically treated due to a severe skeletal class III malocclusion. No difference in facial symmetry was found, but the facial attractiveness of subjects with a cleft was rated as poorer. Although the authors suggested that the visibility of the residual lip scarring present only in patients with the cleft had an influence on facial attractiveness, it is also possible that abnormal nasolabial form, observed in subjects with clefts but not in subjects with class III malocclusion, had a considerable effect on esthetic rating. However, this hypothesis requires further research.

Our findings show that nasolabial asymmetry increased as the severity of the cleft increased. The absolute nasolabial asymmetry in subjects with UCL was significantly less than in subjects with UCLAP. This is likely to have been caused by differences in the bone support of the soft tissues: In UCL, the alveolar bone is intact and the repaired lip is supported by the alveolus in a manner similar to that in subjects without cleft, whereas in UCLAP, there is a bony defect resulting in 
Table 4 Mean esthetic scores (overall and for four nasolabial components) in subjects with UCL, UCLA, and UCLAP

\begin{tabular}{llll}
\hline & $\begin{array}{l}\text { UCL } \\
\text { Mean (SE, 95 \% CI) }\end{array}$ & $\begin{array}{l}\text { UCLA } \\
\text { Mean (SE, 95 \% CI) }\end{array}$ & $\begin{array}{l}\text { UCLAP } \\
\text { Mean, (SE, 95 \% CI) }\end{array}$ \\
\hline Overall score & $59.40(2.30,54.27$ to 64.53$)$ & $56.27(2.00,52.17$ to 60.36$)$ & $50.5,(1.32,47.85$ to 53.20$)$ \\
Nose shape & $61.78(2.27,56.74$ to 66.83$)$ & $57.47(2.62,52.10$ to 62.83$)$ & $51.17(1.90,47.34$ to 55.00$)$ \\
Nose deviation & $61.67(2.48,56.15$ to 67.19$)$ & $59.01(2.20,54.50$ to 63.51$)$ & $52.85(1.46,49.91$ to 55.79$)$ \\
Vermilion border & $56.89(2.27,51.83$ to 61.94$)$ & $55.41(2.25,50.80$ to 60.02$)$ & $50.70(1.48,47.70$ to 53.70$)$ \\
Profile & $57.26(3.54,49.38$ to 65.14$)$ & $53.18(2.49,48.10$ to 58.29$)$ & $47.37(1.96,43.41$ to 51.34$)$ \\
\hline
\end{tabular}

$S E$, standard error; $C I$, confidence interval; $U C L$, unilateral cleft lip; $U C L A$, unilateral cleft lip and alveolus; $U C L A P$, unilateral cleft lip, alveolus, and palate

poor labial support and hence increased asymmetry. Other authors have reported similar results [37-39].

To date, research on human facial attractiveness has been done mostly with the use of frontal 2D images. However, our primary visual experience with faces is in three dimensions. Moreover, perception of the face in the human visual system is viewpoint specific, i.e., esthetic judgment based on one viewpoint may not be generalizable to other viewpoints [40]. The use of 3D images may overcome this problem, because manipulation of $3 \mathrm{D}$ photographs allows facial esthetics to be rated from all viewpoints. Indeed, Tigue et al. showed that application of $3 \mathrm{D}$ stimulus media enhanced the ability of raters to assess facial attractiveness [40]. This could indicate that the more information retrieved from the stimulus medium and processed during rating of facial esthetics, the more accurate the esthetic assessment.

In this study, we used a modified Asher-McDade's esthetic index, with a $0-100$ VAS in place of a five-point scale. The advantage of the VAS is that biases toward preferred values that are found when using categorical or equal-appearing interval scales are avoided [41, 42]. Furthermore, the use of
VAS produces more objective reproducible and reliable results [43]; VAS is also more sensitive [41, 42] than categorical rating scales with or without verbal descriptors [32, 44]. However, the disadvantage of VAS is that the obtained numeric values cannot be connected with certain categories or ordered scales, because the data are usually not normally distributed $[45,46]$.

The composition of the rating panel can influence the outcome of evaluation of facial appearance in subjects with a cleft. Previous studies showed that rating panels comprising laypersons were more critical [32, 47, 48], less critical $[49,50]$, or equally critical [51] to rating panels consisting of professionals (i.e., persons involved in the treatment of cleft lip and palate). These contradictory findings do not imply that rating facial esthetics is unfeasible because of a high risk of inconsistent results. In fact, the variation in scores assigned by various panels indicates that some panels are more critical and some are less critical than others. Nevertheless, there is strong evidence that if panels are asked to rank facial attractiveness (i.e., to order from least to most attractive), the obtained rankings are likely to be comparable irrespective of the panel composition [4]. Therefore, the

Table 5 Inter-group differences in esthetic scores (overall and for four nasolabial components) among subjects with UCL, UCLA, and UCLAP assessed with ANOVA and post hoc Tukey tests

\begin{tabular}{|c|c|c|c|c|c|c|}
\hline & \multicolumn{2}{|l|}{ UCL vs. UCLA } & \multicolumn{2}{|l|}{ UCL vs. UCLAP } & \multicolumn{2}{|c|}{ UCLA vs. UCLAP } \\
\hline & $\begin{array}{l}\text { Mean difference } \\
\text { (SE) }\end{array}$ & $p(95 \% \mathrm{CI})$ & $\begin{array}{l}\text { Mean difference } \\
\text { (SE) }\end{array}$ & $p(95 \% \mathrm{CI})$ & $\begin{array}{l}\text { Mean difference } \\
\text { (SE) }\end{array}$ & $p(95 \% \mathrm{CI})$ \\
\hline Overall score & $3.13(3.33)$ & $0.617(-4.82$ to 11.09$)$ & $8.88(3.19)$ & 0.018 (1.25 to 16.50$)$ & $5.74(2.25)$ & $0.033(0.38$ to 11.11$)$ \\
\hline Nose shape & $4.32(4.47)$ & $0.601(-6.36$ to 15.00$)$ & $10.61(4.29)$ & $0.040(0.04$ to 20.85$)$ & $6.30(3.02)$ & $0.099(-0.91$ to 13.50$)$ \\
\hline Nose deviation & $2.66(3.66)$ & $0.748(-6.08$ to 11.40$)$ & $8.82(3.51)$ & $0.037(0.44$ to 17.20$)$ & $6.16(2.47)$ & 0.039 (0.26 to 12.06) \\
\hline Vermilion border & $1.47(3.71)$ & $0.917(-7.39$ to 10.34$)$ & $6.19(3.56)$ & $0.197(-2.31$ to 14.68$)$ & $4.71(2.51)$ & $0.151(-1.27$ to 10.69$)$ \\
\hline Profile & $4.07(4.59)$ & $0.650(-6.89$ to 15.04$)$ & $9.88(4.40)$ & $0.070(-0.63$ to 20.40$)$ & $5.81(3.10)$ & $0.153(-1.59$ to 13.21$)$ \\
\hline
\end{tabular}

$S E$, standard error; $C I$, confidence interval; $U C L$, unilateral cleft lip; $U C L A$, unilateral cleft lip and alveolus; $U C L A P$, unilateral cleft lip, alveolus, and palate

Bold font denotes statistical significance 
Table 6 Results of regression analyses with overall esthetics and esthetics of four nasolabial components as dependent variables and absolute nasolabial asymmetry as independent variable

\begin{tabular}{|c|c|c|c|c|}
\hline Nasolabial component & Effect of nasolabial asymmetry & $p$ & $95 \% \mathrm{CI}$ & $R^{2}$ \\
\hline Overall esthetics & -9.61 & $<0.001$ & $(-14.04$ to -5.19$)$ & 0.19 \\
\hline Nose shape & -9.72 & 0.002 & $(-15.83$ to -3.61$)$ & 0.11 \\
\hline Nose deviation & -12.08 & $<0.001$ & $(-16.72$ to -7.44$)$ & 0.25 \\
\hline Vermilion border & -8.53 & 0.001 & $(-13.46$ to -3.59$)$ & 0.13 \\
\hline Profile view & -8.12 & 0.013 & $(-14.47$ to -1.78$)$ & 0.73 \\
\hline
\end{tabular}

Subjects with various cleft types (UCL, UCLA, and UCLAP) were pooled together. Bold font denotes statistical significance

$C I$, confidence interval; $U C L$, unilateral cleft lip; UCLA, unilateral cleft lip and alveolus; UCLAP, unilateral cleft lip, alveolus, and palate

choice of the rating panel can be based on issues such as the research question or the availability of judges.

In the current investigation, we evaluated nasolabial symmetry based on all data points of the 3D facial surface. This is in contrast with most previous works, in which single soft-tissue landmarks were used to determine facial asymmetry. Our method allows for a more global and precise evaluation of the facial surface. Additionally, the standardized procedure that we utilized does not require any manual definition of single landmarks and is believed to result in better inter-observer reliability [52]. The high degree of reproducibility and validity described in previous studies [53-55], along with the absence of relevant systematic errors in the method, implies that the technique of determination of facial asymmetry presented herein is appropriate and can be recommended for future research.

In summary, a starting point of this study was a search for factors affecting nasolabial appearance in
CLP. We also planned to clarify the role of nasolabial symmetry in perception of facial esthetics. We were surprised that only few studies [23-27] dealt with the relationship between symmetry and esthetics in CLP (and none used 3D imaging). Moreover, their findings were equivocal. We suspected that this paucity of publications might have resulted from prevailing opinion that "what is symmetrical is more beautiful" and most researchers just did not bother to confirm the obvious. However, our clinical impression was that in more severe forms of CLP, the nasolabial symmetry was less important than the shape of this region for esthetic judgment. Our findings seem to confirm our impression - the more severe the cleft, the less important the symmetry is for perception of nasolabial appearance. Thus, this study demonstrates for the first time that using nasolabial symmetry as a main measure of quality of treatment of severe forms of CLP is not sufficient and may be unrelated with patient's perception of facial appearance.
Table 7 Results of regression analyses with esthetics of nasolabial components as dependent variables and absolute nasolabial asymmetry as independent variable in groups with various cleft types (UCL, UCLA, and UCLAP)

Bold font denotes statistical significance

$C I$, confidence interval; $U C L$ unilateral cleft lip; UCLA, unilateral cleft lip and alveolus; $U C L A P$, unilateral cleft lip, alveolus, and palate

\begin{tabular}{llllll}
\hline $\begin{array}{l}\text { Nasolabial } \\
\text { component }\end{array}$ & $\begin{array}{l}\text { Cleft type } \\
\text { group }\end{array}$ & $\begin{array}{l}\text { Effect of } \\
\text { nasolabial } \\
\text { asymmetry }\end{array}$ & $p$ & $95 \%$ CI & $R^{2}$ \\
\hline Overall esthetics & UCL & -14.814 & $\mathbf{0 . 0 0 8}$ & $(-24.78$ to -4.85$)$ & 0.56 \\
& UCLA & -13.837 & $\mathbf{0 . 0 0 2}$ & $(-22.32$ to -5.35$)$ & 0.29 \\
Nose shape & UCLAP & -3.858 & 0.199 & $(-9.83$ to 2.11$)$ & 0.04 \\
& UCL & -13.604 & $\mathbf{0 . 0 1 7}$ & $(-24.17$ to -3.04$)$ & 0.49 \\
Nose deviation & UCLA & -18.228 & $\mathbf{0 . 0 0 2}$ & $(-29.32$ to -7.13$)$ & 0.29 \\
& UCLAP & -0.874 & 0.841 & $(-9.60$ to 7.85$)$ & 0.01 \\
Vermilion border & UCL & -17.757 & $\mathbf{0 . 0 0 2}$ & $(-26.70$ to -8.82$)$ & 0.70 \\
& UCLA & -21.325 & $<\mathbf{0 . 0 0 1}$ & $(-28.65$ to -14.00$)$ & 0.56 \\
& UCLAP & -3.577 & 0.281 & $(-10.19$ to 3.04$)$ & 0.03 \\
Profile view & UCLA & -9.050 & 0.152 & $(-22.12$ to 4.02$)$ & 0.21 \\
& UCLAP & -7.994 & 0.143 & $(-18.86$ to 2.87$)$ & 0.08 \\
& UCL & -18.843 & $\mathbf{0 . 0 2 4}$ & $(-13.88$ to -1.01$)$ & 0.12 \\
& UCLA & -7.799 & $\mathbf{0 . 0 4 3}$ & $(-36.93$ to -0.76$)$ & 0.38 \\
& UCLAP & -3.540 & 0.198 & $(-19.92$ to 4.32$)$ & 0.06 \\
& & & 0.430 & $(-12.51$ to 5.43$)$ & 0.02 \\
\hline
\end{tabular}




\section{Conclusions}

Based on our findings, we conclude that, overall, the perception of nasolabial esthetics is influenced by nasolabial symmetry. As a result, objectively quantified nasolabial symmetry can be used as a measure of outcome of the treatment of cleft lip and palate. However, in patients with unilateral cleft lip, alveolus, and palate, factors other than nasolabial asymmetry can influence the rating of nasolabial esthetics.

Conflict of interest The authors report no conflict of interest.

\section{References}

1. Aharon I, Etcoff N, Ariely D, Chabris CF, O'Connor E et al (2001) Beautiful faces have variable reward value: fMRI and behavioral evidence. Neuron 32:537-551

2. O’Doherty J, Winston J, Critchley H, Perrett D, Burt DM et al (2003) Beauty in a smile: the role of medial orbitofrontal cortex in facial attractiveness. Neuropsychologia 41:147-155

3. Dion K, Walster E, Berschei E (1972) What is beautiful is good. J Pers Soc Psychol 24:285

4. Langlois JH, Kalakanis L, Rubenstein AJ, Larson A, Hallam M et al (2000) Maxims or myths of beauty? A meta-analytic and theoretical review. Psychol Bull 126:390-423

5. Eagly AH, Makhijani MG, Ashmore RD, Longo LC (1991) What is beautiful is good, but - a meta-analytic review of research on the physical attractiveness stereotype. Psychol Bull 110:109-128

6. Benson PL, Karabenick SA, Lerner RM (1976) Pretty pleases - effects of physical attractiveness, race, and sex on receiving help. J Exp Soc Psychol 12:409-415

7. Ritts V, Patterson ML, Tubbs ME (1992) Expectations, impressions, and judgments of physically attractive students - a review. Rev Educ Res 62:413-426

8. Rhodes G (2006) The evolutionary psychology of facial beauty. Annu Rev Psychol 57:199-226

9. Cunningham MR, Roberts AR, Wu CH, Barbee AP, Druen PB (1995) Their ideas of beauty are, on the whole, the same as oursconsistency and variability in the cross-cultural perception of female physical attractiveness. J Pers Soc Psychol 68:261-279

10. Perrett DI, May KA, Yoshikawa S (1994) Facial shape and judgments of female attractiveness. Nature 368:239-242

11. Rhodes G, Yoshikawa S, Clark A, Lee K, McKay R et al (2001) Attractiveness of facial averageness and symmetry in non-Western cultures: in search of biologically based standards of beauty. Perception 30:611-625

12. Geldart S, Maurer D, Carney K (1999) Effects of eye size on adults' aesthetic ratings of faces and 5-month-olds' looking times. Perception 28:361-374

13. Langlois JH, Roggman LA, Casey RJ, Ritter JM, Rieserdanner LA et al (1987) Infant preferences for attractive faces - rudiments of a stereotype. Dev Psychol 23:363-369

14. Rubenstein AJ, Kalakanis L, Langlois JH (1999) Infant preferences for attractive faces: a cognitive explanation. Dev Psychol 35:848-855

15. Samuels CA, Butterworth G, Roberts T, Graupner L, Hole G (1994) Facial aesthetics - babies prefer attractiveness to symmetry. Perception 23:823-831

16. Samuels CA, Ewy R (1985) Aesthetic perception of faces during infancy. Br J Dev Psychol 3:221-228
17. Slater A, Quinn PC, Hayes R, Brown E (2000) The role of facial orientation in newborn infants' preference for attractive faces. Dev Sci 3:181-185

18. Enquist M, Arak A (1994) Symmetry, beauty and evolution. Nature 372:169-172

19. Jansson L, Forkman B, Enquist M (2002) Experimental evidence of receiver bias for symmetry. Anim Behav 63:617-621

20. Johnstone RA (1994) Female preference for symmetrical males as a by-product of selection for mate recognition. Nature 372:172-175

21. Perrett DI, Burt DM, Penton-Voak IS, Lee KJ, Rowland DA et al (1999) Symmetry and human facial attractiveness. Evol Hum Behav 20:295-307

22. Rhodes G, Sumich A, Byatt G (1999) Are average facial configurations attractive only because of their symmetry? Psychol Sci 10:5258

23. Russell KA, Waldman SD, Tompson B, Lee JM (2001) Nasal morphology and shape parameters as predictors of nasal esthetics in individuals with complete unilateral cleft lip and palate. Cleft Palate Craniofac J J38:476-485

24. Fudalej P, Katsaros C, Hozyasz K, Borstlap WA, Kuijpers-Jagtman AM (2012) Nasolabial symmetry and aesthetics in children with complete unilateral cleft lip and palate. Br J Oral Maxillofac Surg 50:621-625

25. Coghlan BA, Matthews B, Pigott RW (1987) A computer-based method of measuring facial asymmetry. Results from an assessment of the repair of cleft lip deformities. Br J Plast Surg 40:371-376

26. Russell KA, Tompson B (2009) Correlation between facial morphology and esthetics in patients with repaired complete unilateral cleft lip and palate. Cleft Palate Craniofac J J46:319-325

27. Robertsharry DP, Evans R, Hathorn IS (1991) Effects of different surgical regimes on nasal asymmetry and facial attractiveness in patients with clefts of the lip and palate. Cleft Palate Craniofac J J28: 274-278

28. Verhoeven TJ, Coppen C, Barkhuysen R, Bronkhorst EM, Merkx MA et al (2013) Three dimensional evaluation of facial asymmetry after mandibular reconstruction: validation of a new method using stereophotogrammetry. Int J Oral Maxillofac Surg 42:19-25

29. Asher-McDade C, Roberts C, Shaw WC, Gallager C (1991) Development of a method for rating nasolabial appearance in patients with clefts of the lip and palate. Cleft Palate Craniofac J 28:385-390

30. Asher-McDade C, Brattstrom V, Dahl E, McWilliam J, Molsted K et al (1992) A six-center international study of treatment outcome in patients with clefts of the lip and palate: Part 4. Assessment of nasolabial appearance. Cleft Palate Craniofac J 29:409-412

31. Schmitt N (1996) Uses and abuses of coefficient alpha. Psychol Assess 8:350-353

32. Papamanou DA, Gkantidis N, Topouzelis N, Christou P (2012) Appreciation of cleft lip and palate treatment outcome by professionals and laypeople. Eur J Orthod 34:553-560

33. Hunt O, Burden D, Hepper P, Johnston C (2005) The psychosocial effects of cleft lip and palate: a systematic review. Eur J Orthod 27: 274-285

34. Meyer-Marcotty P, Alpers GW, Gerdes ABM, Stellzig-Eisenhauer A (2010) Impact of facial asymmetry in visual perception: a 3dimensional data analysis. Am J Orthod Dentofacial Orthop 137: 168.e1-8

35. Fudalej P, Katsaros C, Bongaarts C, Dudkiewicz Z, KuijpersJagtman AM (2009) Nasolabial esthetics in children with complete unilateral cleft lip and palate after 1- versus 3-stage treatment protocols. J Oral Maxillofac Surg 67:1661-1666

36. Mercado A, Russell K, Hathaway R, Daskalogiannakis J, Sadek H et al (2011) The Americleft study: an inter-center study of treatment outcomes for patients with unilateral cleft lip and palate part 4 . Nasolabial aesthetics. Cleft Palate Craniofac J 48:259-264 
37. Hood CA, Bock M, Hosey MT, Bowman A, Ayoub AF (2003) Facial asymmetry - 3D assessment of infants with cleft lip \& palate. Int J Paediatr Dent 13:404-410

38. Bugaighis I, O'Higgins P, Tiddeman B, Mattick C, Ben Ali O et al (2010) Three-dimensional geometric morphometrics applied to the study of children with cleft lip and/or palate from the North East of England. Eur J Orthod 32:514-521

39. Bell A, Lo TW, Brown D, Bowman AW, Siebert JP, et al (2013) Three-dimensional assessment of facial appearance following surgical repair of unilateral cleft lip and palate. Cleft Palate Craniofac J

40. Tigue CC, Pisanski K, O'Connor JJM, Fraccaro PJ, Feinberg DR (2012) Men's judgments of women's facial attractiveness from twoand three-dimensional images are similar. J VisNov 6;12(12). doi:pii: 3. $10.1167 / 12.12 .3$

41. Howells DJ, Shaw WC (1985) The validity and reliability of ratings of dental and facial attractiveness for epidemiologic use. Am J Orthod Dentofacial Orthop 88:402-408

42. Grant S, Aitchison T, Henderson E, Christie J, Zare S et al (1999) A comparison of the reproducibility and the sensitivity to change of visual analogue scales, Borg scales, and Likert scales in normal subjects during submaximal exercise. Chest 116:1208-1217

43. Grossman SA, Sheidler VR, Mcguire DB, Geer C, Santor D et al (1992) A comparison of the hopkins pain rating instrument with standard visual analog and verbal descriptor scales in patients with cancer pain. J Pain Symptom Manage 7:196-203

44. Williamson A, Hoggart B (2005) Pain: a review of three commonly used pain rating scales. J Clin Nurs 14:798-804

45. Svensson E (2000) Concordance between ratings using different scales for the same variable. Stat Med 19:3483-3496

46. Knutsson I, Rydstrom H, Reimer J, Nyberg P, Hagell P (2010) Interpretation of response categories in patient-reported rating scales: a controlled study among people with Parkinson's disease. Health Qual Life Outcomes 8:61
47. Eliason MJ, Hardin MA, Olin WH (1991) Factors that influence ratings of facial appearance for children with cleft-lip and palate. Cleft Palate Craniofac J J28:190-193

48. Gkantidis N, Papamanou DA, Christou P, Topouzelis N (2013) Aesthetic outcome of cleft lip and palate treatment. Perceptions of patients, families, and health professionals compared to the general public. J Craniomaxillofac Surg 41:e105-e110

49. Mani MR, Semb G, Andlin-Sobocki A (2010) Nasolabial appearance in adults with repaired unilateral cleft lip and palate: relation between professional and lay rating and patients' satisfaction. J Plast Surg Hand Surg 44:191-198

50. Halberstadt J, Rhodes G (2003) It's not just average faces that are attractive: computer-manipulated averageness makes birds, fish, and automobiles attractive. Psychon Bull Rev 10:149-156

51. Bongaarts CAM, Prahl-Andersen B, Bronkhorst EM, Spauwen PHM, Mulder JW et al (2008) Effect of infant orthopedics on facial appearance of toddlers with complete unilateral cleft lip and palate (Dutchcleft). Cleft Palate Craniofac J J45:407-413

52. Meyer-Marcotty P, Kochel J, Boehm H, Linz C, Klammert U et al (2011) Face perception in patients with unilateral cleft lip and palate and patients with severe Class III malocclusion compared to controls. J Craniomaxillofac Surg 39:158-163

53. Benz MLX, Maier T, Nkenke E, Seeger S, Neukam FW, Hausler G (2002) Vision, modeling, and visualization; Greiner G NH, Ertl T, Girod B, Seidel HP, editor. IOS Press, Amsterdam, pp 332-339

54. Nkenke E, Langer A, Laboureux X, Benz M, Maier T et al (2003) Validation of in vivo assessment of facial soft-tissue volume changes and clinical application in midfacial distraction: a technical report. Plast Reconstr Surg 112:367-380

55. Hartmann J, Meyer-Marcotty P, Benz M, Haeusler G, StellzigEisenhauer A (2007) Reliability of a method for computing facial symmetry plane and degree of asymmetry based on $3 \mathrm{D}$-data. $\mathrm{J}$ Orofac Orthop 68:477-490 